\title{
Registros Precodificados \\ para Parto Hospitalario, 3.136 Casos
}

\author{
Dr. Carlos Alberto Esparza Duque* \\ Dr. Mario Calle Mesa** \\ Universidad de Caldas - Facultad de Medicina \\ Hospital Universitario de Caldas Departamento de Ginecología y Obstetricia
}

\section{INTRODUCCION}

En 1968, L.J. Meléndez, de la Universidad Javeriana, publicó en Universitas Médica, un trabajo intitulado "Historias Clínicas Funcionales" (1). Sus críticas a la historia clínica tradicional continúan hoy en plena vigencia, porque ese mismo tipo de historia es el que se sigue empleando en los hospitales del país.

El Ministerio de Salud, en un documento titulado "Conceptos Generales. Administración de Registros Médicos y Modelo de Historia Clínica Básica", propone una solución dentro del contexto del Sistema Nacional de Salud y los conceptos de ciudado progresivo y atención integral (2).

Pensamos que tanto la historia clínica funcional propuesta por Meléndez e inspirada en los trabajos de L.L. Weed, como el proyecto del Ministerio de Sa-

\footnotetext{
* Profesor Asociado

* Jefe del Departamento
}

lud, se quedan cortos, a la luz de los progresos cada día más acelerados de la microelectrónica y de la informática.

En 1981 es necesario pensar en la historia clínica computarizada, como la solución más completa y moderna al problema médico fundamental de las histo rias clínicas, con profundas repercusiones en los campos asistencial, docente, investigativo, administrativo y de planeación de la salud.

Desde hace una década, los autores hemos tenido preocupación por la pésima calidad de nuestras historias clínicas en general y por las gineco-obstétricas en particular. El año pasado uno de nosotros (MCM), decidió presentar un proyecto de historias precodificadas, que substituyan a las tradicionales. Lo más importante de ellas, son los principios en que se fundan. La forma es susceptible de muchas modificaciones.

El presente estudio es resultado de la aplicación del modelo de historia pre- 
codificada para parto y puerperio hospitalario durante un año.

Los propósitos del estudio son los siguientes:

1. Presentar un tipo moderno de historia o registro clínico que permite procesar la información en computador.

2. Analizar las características de la historia, sus limitaciones y sugerir desarrollos futuros.

3. Exponer solamente algunos de los resultados de carácter descriptivo del estudio.

4. Fomentar el interés por las historias clínicas computarizadas, dentro del cuerpo médico.

\section{Material y Métodos}

Se hace un estudio de 3.136 registros precodificados de parto y puerperio, correspondientes a pacientes admitidas al Servicio de Obstetricia y Ginecología, entre el 1o. de julio de 1980 y el 30 de junio de 1981.

La información se registró en un cuestionario precodificado de 2 páginas. En la primera página es posible registrar 51 variables y más de 171 códigos de las mismas. Lo único que el médico necesita escribir, son numeros arábigos y círculos.

El formulario irá acompañado en el futuro de un manual de instrucciones en el cual se definen con precisión los términos con el fin de que quienes lo diligencien, lo hagan con criterio unificado. Para la presente investigación se dieron instrucciones, tan precisas como fue posible, a quienes colaboraron en la recolección de los datos.

Los formularios fueron revisados personalmente por uno de los autores
(MCM) y posteriormente se remitieron a la Corporación Centro Regional de Población (CCRP), en donde la información fue transferida a tarjetas perforadas, debidamente verificada. Luego se procesó en computador.

De esta manera se obtuvieron las distribuciones de frecuencia uni y bivariadas que constituyen el material que parcialmente se analiza en el presente estudio.

No es posible en un trabajo como éste analizar exahustivamente toda la gran cantidad de información suministrada por el computador, que comprende en total 82 tablas.

Se estudian las distribuciones de frecuencias relativas a los siguientes aspectos:

Zona de residencia
Distribución por edades
Escolaridad en años cursados
Estado Civil
Número de hijos nacidos vivos
Número de abortos previos
Número de hijos actualmente vivos
Patología en el embarazo anterior
Resultado del último embarazo
Forma de terminación del último
parto
Número de controles prenatales
Número de cigarrillos/día consumidos
durante el embarazo
Cesáreas previas
Peso de las pacientes
Talla de las pacientes
Duración del embarazo en semanas
cumplidas
Duración en horas de la ruptura de
membranas anteparto
Tipo de trabajo de parto
Tipo de presentación durante el parto
Anestesia empleada
Tipo de episiotomía
Tipo de parto
Complicaciones del parto


Persona que atendió el parto

Peso del recién nacido

Sexo del recién nacido

Indice de apgar al minuto

Indice de apgar a los 5 minutos

Estado del recién nacido

Complicaciones del puerperio

Se obtuvieron 35 tablas que correlacionan diferentes variables, cuyo análisis se presentará en otro u otros estudios.

\section{Resultados.}

El $81.0 \%$ de las pacientes pertenecen al área urbana, en su gran mayoría $(75.8 \%)$ de Manizales.

Tabla No. 1

\section{DISTRIBUCION POR EDADES}

\begin{tabular}{lrr}
\hline Grupos de edad & Número & Porcentaje \\
\hline $12-15$ & 41 & 1.3 \\
$16-19$ & 756 & 24.2 \\
$20-24$ & 1.138 & 36.4 \\
$25-29$ & 638 & 20.4 \\
$30-34$ & 333 & 10.7 \\
$35-39$ & 160 & 5.2 \\
$40-44$ & 54 & 1.7 \\
45 o más & 2 & 0.1 \\
\hline Total & 3.122 & 100.0 \\
\hline
\end{tabular}

Promedio: 23.9 años
D/S.
5.9
Rango:
12 a 47 años

El $25.5 \%$ corresponde a mujeres de 12 a 19 años. Solamente hay un $7 \%$ de 35 ó más años.

En cuanto a escolaridad encontramos un $6.4 \%$ de analfabetismo y $39.1 \%$ que solo habían cursado primaria incom- pleta. El promedio de años de estudio en este grupo fue de 2.7, para secundaria incompleta el promedio es 2.4 . Con respecto a estado civil el $41.2 \%$ eran solteras o en unión libre, con un promedio absoluto del primer grupo.

En los antecedentes obstétricos encontramos los datos siguientes:

\section{Paridad promedio 2.6}

El promedio de hijos nacidos vivos para todo el grupo fue de 1.4 con una D.S. de 2.0. Eliminando las primigestantes obtuvimos la tabla siguiente:

Tabla No. 2

NACIDOS VIVOS

\begin{tabular}{lrr}
\hline & Número & Porcentaje \\
\hline Ninguno & 83 & 4.4 \\
$1-3$ & 1.430 & 75.7 \\
$4-6$ & 263 & 13.9 \\
$7-9$ & 81 & 4.3 \\
10 o más & 31 & 1.7 \\
\hline Total & 1.888 & 100.0 \\
\hline
\end{tabular}

Promedio: 2.5

El promedio de hijos actualmente vivos para todo el grupo fue de 1.2 con D.S. de 1.7 .

No teniendo en consideración a las primigestantes, resulta la Tabla No. 3 .

El promedio de abortos, excluyendo a las primigestantes, fue de 1.5 .

En cuanto a patología en embarazos anteriores hay 99 códigos correspondientes a otras tantas entidades. Excluimos 
Tabla No. 3

HIJOS ACTUALMENTE VIVOS

\begin{tabular}{lrr}
\hline & Número & Porcentaje \\
\hline Ninguno & 129 & 6.8 \\
$1-3$ & 1.446 & 76.8 \\
$4-6$ & 237 & 12.6 \\
7 o más & 72 & 3.8 \\
\hline Total & 1.884 & 100.0 \\
\hline
\end{tabular}

\section{PROMEDIO 2.3}

a las primigestantes (1.246) para el cálculo de las estadísticas y mencionamos sólo algunas de las más importantes:

\begin{tabular}{ll} 
Amenaza de aborto & $2.5 \%$ \\
Preeclampsia-eclampsia & $1.4 \%$ \\
Pielonefritis & $1.0 \%$ \\
Prematurez & $0.6 \%$ \\
Otra patología & $99 \%$ \\
\multicolumn{2}{c}{ Tabla No. 4} \\
RESULTADO DEL ULTIMO \\
EMBARAZO
\end{tabular}

Número Porcentaje

\begin{tabular}{lrr} 
Vivo a término & 1.582 & 83.7 \\
Vivo prematuro & 62 & 3.3 \\
Mortinato & 20 & 1.0 \\
Aborto & 217 & 11.5 \\
Ectópico & 1 & 0.1 \\
Otro & 8 & 0.4 \\
\hline Total & 1.890 & 100.0 \\
\hline
\end{tabular}

El 83.7\% fueron partos normales.

El $11.5 \%$ o abortos
Tabla No. 5

FORMA DE TERMINACION DEL PARTO ANTERIOR

Número Porcentaje

$\begin{array}{lrr}\text { Normal } & 1.505 & 90.0 \\ \text { Inducido } & 25 & 1.5 \\ \text { Fórceps } & 16 & 0.9 \\ \text { Vacum } & 1 & 0.1 \\ \text { Cesárea } & 105 & 6.3 \\ \text { En pelvis } & 10 & 0.6 \\ \text { Versión interna } & 1 & 0.1 \\ \text { Otro } & 8 & 0.5\end{array}$

$\begin{array}{lll}\text { Total } & 1.671 & 100.0\end{array}$

$90.0 \%$ habían sido partos normales, $6.3 \%$ terminaron por cesárea.

No había tenido control prenatal el $35.2 \%$. El promedio de controles fue de 3.0 con D.S. de 2.8. Eran fumadoras el $24.7 \%$. De las pacientes que habían tenido cesáreas previas, el 83.2 había tenido una sola y el $16.1 \% 2$.

La duración promedio del embarazo en semanas fue de 38.7. Altura uterina promedio: 31.9 con D.S. de 2.8. El $68.1 \%$ tenía membranas íntegras en el momento del ingreso. Se hizo amniotomía en $12.8 \%$ de los partos. Fueron inducidos el 2.8, siendo el método más empleado el goteo simple con oxitocina $(2.0 \%)$.

Sobre el tipo de presentación obtuvimos los siguientes resultados: cefálica $96.2 \%$; pelvis $3.3 \%$; hombro $0.5 \%$.

No se empleó anestesia en el $34.2 \%$. Anestesia local para episiotomías en el 
Tabia No. 6

PESO DE LAS PACIENTES

\begin{tabular}{lrr}
\hline Peso en Kg. & Número & Porcentaje \\
\hline 49 o menos & 178 & 7.7 \\
$50-59$ & 907 & 39.5 \\
$60-69$ & 879 & 38.2 \\
$70-79$ & 272 & 11.8 \\
$80-89$ & 50 & 2.1 \\
90 o más & 15 & 0.7 \\
\hline Total & & \\
\hline
\end{tabular}

Peso promedio: $60.5 \mathrm{Kg}$.

D. S. : 8.8

Rango : 40 a 97 Kgs.

Tabla No. 7

TALLA DE LAS PACIENTES

Talla en $\mathrm{Cm}$. Número Porcentaje

\begin{tabular}{lrr}
\hline 139 o menos & 36 & 1.6 \\
$140-149$ & 755 & 32.8 \\
$150-159$ & 1.263 & 54.9 \\
$160-169$ & 234 & 10.2 \\
$170-179$ & 9 & 0.4 \\
180 o más & 3 & 0.1 \\
\hline Total & 2.300 & 100.0 \\
\hline
\end{tabular}

Promedio: 151.8

D. S. : 6.3

Rango : 104 a 184

$50.5 \%$. Conductiva $8.7 \%$ y general $5.1 \%$. Estas dos últimas para cesáreas.
Episiotomía mediana $27.8 \%$. Mediolateral 23.2.

$15.6 \%$ de los partos tuvieron complicaciones, siendo las más frecuentes la desproporción fetopélvica, el trabajo de parto disfuncional, la retención de placenta y la corioamnionitis.

El $71.5 \%$ de los partos fue atendido por Médicos Internos. El $20.1 \%$ por Médicos Residentes o Especialistas.

Tabla No. 8

PESO DEL RECIEN NACIDO

\begin{tabular}{lrr}
\hline Peso en gramos & Número & Porcentaje \\
\hline $1.000-1.499$ & 19 & 0.6 \\
$1.500-1.999$ & 36 & 1.2 \\
$2.000-2.499$ & 206 & 6.6 \\
$2.500-2.999$ & 843 & 27.0 \\
$3.000-3.499$ & 1.294 & 41.5 \\
$3.500-3.999$ & 630 & 20.2 \\
$4.000-4.499$ & 84 & 2.7 \\
$4.500-$ o más & 7 & 0.2 \\
\hline Total & 3.119 & 100.0 \\
\hline
\end{tabular}

Peso Promedio: 3.114 .5 gms.

D. S. $\quad$ : $\quad 486.25 \mathrm{gms}$.

Rango : 1.060 a 4.820 gms.

En cuanto a sexo de los recién nacidos encontramos que $54,1 \%$ eran mujeres y $45.9 \%$ o varones.

El índice de Apgar al minuto fue de 7 ó más en $86.8 \%$ de los casos, y en $97.8 \%$ a los 5 minutos. Severamente deprimidos $2.8 \%$ al minuto y $2.2 \%$ a los cinco minutos. 
El $86.3 \%$ de los recién nacidos fue normal. Ocurrió sufrimiento fetal en $7.3 \%$. Malformaciones congénitas en$1.7 \%$. Traumatismo en $0.2 \%$.

Entre las complicaciones más frecuentes del puerperio encontramos hemorragias en $2.0 \%$, endometritis en $0.8 \%$, infección de heridas en $0.7 \%$ y otras menos comunes.

\section{Comentario}

Hacemos resaltar, ante todo, el valor y las características del tipo de registro precodificado que nos permitió, una vez recolectada la información, procesarla en computador y obtener así, en pocos minutos, una cantidad tan abundante de datos que resulta difícil analizarlos en su totalidad, dentro de los límites de un trabajo como éste.

La sola idea de revisar un número equivalente de historias clínicas tradicionales (3.136) es desalentador por la enorme inversión que exigiría de esfuerzo manual e intelectual y de tiempo (meses o años tal vez), con la seguridad de que no lograríamos tanta información, como la obtenida en pocos minutos, más válida $y$ confiable, mediante el procesamiento electrónico del contenido de una sola página del tipo de registros que estamos considerando.

Más aún, mediante este sistema nos liberamos de la parte más mecánica pero no menos ardua de las investigaciones clínicas, para dedicar mayor tiempo y energía al análisis de los resultados.

La hoja de registro en que se basa este estudio de parto y puerperio en el Hospital Universitario de Caldas, tiene las siguientes características:

\section{Cincuenta y un viariables o aspectos relativos a:}

1.1 Características sociodemográficas. de las pacientes.

\subsection{Antecedentes obstétricos.}

1.3 Información Médico-Obstétrica referente al embarazo, parto y puerperio actuales.

1.4 Información sobre el recién nacido.

2. Cada variable tiene varios códigos específicos, para un total de más de 171.

3. Se elimina hasta donde es posible la necesidad de la escritura manual. Los únicos signos que el médico emplea, son números arábigos y círculos.

4. La información que se busca está plenamente definida y limitada en el formulario.

5. Los términos se definen, con la mayor precisión posible, en un manual disponible para quien diligencia el formulario. Esto da homogeneidad a la información registrada y unidad de criterio.

6. El tiempo empleado en diligenciar cada formulario se acorta con la experiencia adquirida mediante su uso reiterado.

7. No contiene información repetida.

8. Se ha diseñado procurando que la información buscada sea completa y ordenada. El orden evidentemente es siempre constante.

Varias de estas características constituyen respuesta o solución a otros tantos defectos de los registros o historias clínicas tradicionales:

1. Ser manuscritas, generalmente ilegible e ininteligibles. 
2. Contener información muy heterogénea, según la experiencia, los criterios y otros factores personales de cada médico.

3. La cantidad de información registrada varía desde un renglón a varias páginas, de concisión extrema a prolijidad supérflua.

4. Carentes de sistematización en el registro, porque cada médico tiene su propio sistema.

5. Ser innecesariamente repetitivas de los mismos datos en páginas sucesivas.

6. La recuperación de la información es generalmente muy laboriosa.

Hemos hallado algunas limitaciones y fallas que nos disponemos a subsanar:

1. Falta el manual para diligenciar correctamente el formulario.

2. Hay códigos que si no se especifican sistemáticamente aportan muy poca información utilizable, como por ejemplo los destinados a COMBINACION y OTROS.

3. En algunas variables hay códigos poco importantes que pueden sustituirse por otros más representativos.

4. Falta diseñar hojas complementarias en las que se puedan formular planes terapéuticos y educativos del paciente, exámenes complementarios y evolución de la enfermedad.

La meta es la historia clínica computarizada sobre la cual se publican cada día más estudios de investigación en el mundo. El sistema más integral que conocemos es el desarrollado en Vermont, bajo la dirección del Dr. L.L. Weed (3), con el nombre de THE COMPUTERIZED PROBLEM-ORIENTED
MEDICAL INFORMATION SYSTEM (PROMIS), para cuyo conocimiento remitimos a la bibliografía. A este sistema se alude muy de paso en el proyecto de registros médicos publicado por el Ministerio de Salud (2).

En cuanto a nuestros resultados, comparados con los del estudio cooperativo realizado por la CCRP en 1977 (4) en el cual participamos, podemos hacer las siguientes observaciones:

Tabla No. 9

CARACTERISTICAS DE LAS PACIENTES

\begin{tabular}{llll}
\hline CCRP & HUC & HUC \\
1.977 & 1.977 & 1.981 \\
\hline
\end{tabular}

Casadas $\quad 54.2 \% 68.9 \%$ \% $58.3 \%$

De área urbana 98.4 $84.4 \quad 81.0$

$\begin{array}{llll}\text { Analfabetas } & 4.8 & 2.6 & 6.4\end{array}$

$\begin{array}{llll}\text { Escolaridad } \bar{x} & 5.2 & 4.1 & 4.8\end{array}$

Tabla No. 11

ANTECEDENTES OBSTETRICOS CONTROL PRENATAL

\begin{tabular}{lccc}
\hline & CCRP & HUC & HUC \\
& 1.977 & 1.977 & 1.981 \\
\hline Ultimo embarazo con & & & \\
neonato vivo a térmi- & & & \\
no & $86.0 \% / 0$ & $78.10 \%$ & $83.7 \%$ o \\
Mortinato previo & 4.3 & 3.0 & 1.3 \\
Sin control prenatal & 30.9 & 42.4 & 35.2 \\
Con alguna patología & & & \\
prenatal previa & 24.9 & & 15.4 \\
\hline
\end{tabular}

En la mayoría de las pacientes el embarazo anterior terminó con neonato vivo. El porcentaje menor de mortinatos se encuentra en el estudio actual. Hay también un porcentaje menor de pacientes con patología en el embarazo ante- 
rior que el estudio cooperativo de 1977. Las enfermedades predominantes en la serie que estamos estudiando fueron amenaza de aborto, preeclampsia-eclamp-sia y pielonefritis.

Tabla No. 12

DATOS SOBRE EL PARTO (INTERVENCIONES)

CCRP HUC HUC 1.977. 1.9771 .981

\begin{tabular}{lrrr} 
Anestesia conductiva & $8.9 *$ & 3.7 & 8.7 \\
General & 9.8 & 1.9 & 5.1 \\
Pudenda & 0.4 & 0.7 & 0.1 \\
Episiotomías & 54.4 & 57.4 & 51.1 \\
Fórceps & 7.2 & 3.3 & 3.0 \\
Cesáreas & -7.7 & 5.6 & 13.5 \\
\hline
\end{tabular}

Tanto el uso de anestesia general como de anestesia conductiva ha aumentado en nuestro Hospital, paralelamente al aumento en el porcentaje de cesáreas. Las cifras del CCRP no son estrictamente comparables en estos dos casos pues se refieren no solamente a Hospitales Universitarios sino a todos los demás que participaron en el estudio cooperativo de 1977.

Vale la pena destacar el empleo escaso y decreciente del bloqueo pudendo, que puede aliviar bastante el dolor durante el período expulsivo.
El porcentaje de episiotomías permanece sensiblemente igual.

Pero mientras en 1977 el $44.4 \%$ eran mediolaterales y el $14.0 \%$ medianas, en esta serie $23.3 \%$ son mediolaterales y $27.8 \%$ medianas. Esto pese a que la teoría predica las ventajas de la episiotomía mediana sobre la mediolateral.

El uso de fórceps sigue siendo bajo. En cambio es muy notable el aumento en el porcentaje de cesáreas a más del doble. No hay duda de que el obstetra actual es más experto en cirugía que en aplicación de fórceps.

Tabla No. 13

DATOS SOBRE EL RECIEN NACIDO

\begin{tabular}{llll}
\hline & CCRP & HUC & HUC \\
& 1.977 & 1.977 & 1.981 \\
\hline $\begin{array}{l}\text { Peso } \bar{x} \\
\begin{array}{l}\text { Indice de Apgar al } \\
\text { minuto } \bar{x}\end{array}\end{array}$ & $3.027 \mathrm{~g}$. & $3.041 .4 \mathrm{~g}$. & 3.114 .5 \\
$\begin{array}{l}\text { Indice de Apgar a } \\
\text { los } 5 \text { minutos }\end{array}$ & 7.0 & 7.3 & 7.4 \\
\hline
\end{tabular}

El peso medio del recién nacido ha aumentado ligeramente.

El índice de Apgar de 7 ó más a los cinco minutos en el $97.8 \%$ de los casos es un dato muy alentador en cuanto al resultado inmediato de nuestra atención obstétrica. Nada nos dice en cambio sobre el pronóstico a largo plazo.

\section{PRECODED RECORDS FOR HOSPITAL DELIVERIES \\ 3.136 CASES}

\section{Summary}

Authors propose in this article a model of precoded record for delivery and puerperium which facilitates computer processing. 
A description of its characteristics is made, as a response and solution to the most prominent limitations and drawbacks of traditional medical records, and its own limitations are noted.

On the basis of this model, preliminary research was compared with the results of a 1977 study of a smaller sample in the same hospital, and with those of the cooperative study conducted that same year by CCRP.

The following aspects and data may be of interest for readers: percentage of patients from rural areas, modification of illiteracy rates, percentage of patients at high risk related to their age $(30.8 \%)$, increase in the utilization of general and conductive anesthesia and low use of pudendal block, and notorious increase in the percentage of caesarian sections.

\section{Resumen}

Se propone un modelo de registro precodificado para parto y puerperio, que permite procesar la información en computador.

Se destacan sus características, que constituyen respuesta o solución a muchas de las más graves limitaciones y defectos de los registros tradicionales.

Se anotan sus fallas $y$ limitaciones.

Se hace un comentario breve sobre los resultados del estudio, comparándolos con los obtenidos en 1977 en una muestra pequeña del mismo hospital, y los del estudio cooperativo correspondiente realizado en el mismo año por la CCRP.

Se subrayan los siguientes resultados: porcentaje de pacientes de áreas rurales, aumento del porcentaje de analfabetismo, porcentaje importante $(30.8 \%)$ de pacientes de riesgo mayor por su edad, aumento en el uso de anestesia general y conductiva, incremento notable del porcentaje de cesáreas, empleo escaso del bloqueo pudendo, incongruencia entre teoría y práctica en los tipos de episiotomía empleados.

\section{Referencias}

1. MELENDEZ L.J.: Historias Clínicas Funcionales. De Universitas Médica 10:62, 1968.

2. Conceptos Generales, Administración de Registros Médicos y Modelo de Historia Clínica Básica. Ministerio de Salud. Oficina de Planeación. División de Información, 1978.

3. WEED LL: Medical Records that Guide and Teach. New Eng J. Med. 278: 652, 1968.

4. Atención del Parto Hospitalario en Colombia. Primeros Hallazgos. Primera Edición. Corporación Centro Regional de Población. Bogotá, 1979, pp. 23-52.

\section{Bibliografía}

1. CERUTTI S. TIM'O PIFRI C. A. Method for the Quantification of the Decisionmaking Process in a Computer-Oriented Medical World. Int J. Biomed Comput. 12: 29-57, 1981.

2. SCHENKER WJ. A Menu-driven, Touch Panel Microomputer for Clinical Recordkeeping. Med Instrum 14: 301, 1980.

3. MARGOIS CZ et al. Shuval Automated Problem-oriented Record: An Off-line Record for a Primary Care Clinic. Isr. J. Med Sic 17:170, 1981.

4. BASDEN A. CLARK EM. Data Integrity in a General Practice Computer System (CLINICS) Int J. Biomed Comput 11: $511,1980$.

5. SPARROW J. KUMPEL Z: The Costs Involved in Running a Fully Computeri- 
zed Primary Care System for a District. Med. Inf. (Lond) 5: 181, 1980.

6. McDONALD CJ. WILSON GA, McCABE GP Jr: Physician Response to Computer Reminders. JAMA 244: 1579, 1980.

7. RIENHOFF O. VAN DER WERFF TJ. REICHERTZ PL: Medical Informatics: Developments in West Germany and South Africa. S Afr Med J 58: 30, 1980.

8. STRATMANN WC: Assesing the Problem oriented Approach to care Delivery. Med. Care 18: 456, 1980.

9. GROVES WE, DAVIDSON WR: A Computerized Chart Management System for Medical Records. Comput Programs Biomed 11: 158, 1980.

10. HUGHES SJ: Installing a Computer-based Patient Information System. J. Nurs Adm 10: 7, 1980.

11. WONG HN, TEAGUE CT, CARSON SN: Automated Data Management for Clinicians. A Low-cost Approach. Arch Surg. 115:514, 1980.

12 LAURENT D, MASHRUWALA MD, LU. CAS CP: A Computerized Data-handling System in Hypertension Management. Arch Int Med 140: 345, 1980.

13. McCAMAN B, HIRSH HL: Medical Records: Legal Perspectives Primary Care 6: 681, 1979.

14. EGGERT AA, TRAVER MI, BLANKENHEIM TJ: A Computer-based Record System for a Hospital Transfusion Service. Transfusion 20: 55, 1980.

15. SEITCHIK J. GIBBS CE, MAASKANT BA, CLOGSTON CP: A Computer-based
Obstetric Data Retrieval System. Am J. Obstet Gynecol 135: 562, 1979.

16. GLUCK J: The Computerized Medical Record System: Meeting the Challenge for Nursing. J. Nurs Adm 9: 17, 1979.

17. GIFFORD S, MABERRY D: An Integrated System for Computerized Patient Records. Hosp Community Psychiatry 30: 532, 1979.

18. HIRSH HL: Legal Implications of Patient Records. South Med J 72: 726, 1979.

19. RUTH DH, RIGDEN S, BRUNWORTH D: An Integrated Family-oriented Problem-oriented Medical Record. J. Fam Pract. 8: 1179, 1979.

20. WEED LL: Physicians of the Future. N. Eng. J. Med. 304: 903, 1981.

21. WEED LL: New Premises and New Tools Medical Education. Unpublished.

22. SCHULTZ JR: PROMIS: Problem-oriented Medical Information System. A Medical and Technical Solution to Four Problems in Medicine, PROMIS Laboratory. University of Vermont. Burlington, Vermont 05401. June, 1977.

23. WANNER JF: Wideband Communication System Improves Response Time. PROMIS Laboratory, Burlington, Vermont, 1978.

24. SCHULTZ JR, DAVIS L: The Technology of PROMIS. Proceedings of the IEEE. 67: 1237, 1979.

25. MCNEILL DG: Developing the Complete Computer-Based Information System. J. Nurs Adm November 1979. 